\title{
3 Antibiotika-Resistenzen in der Tierhaltung und im Pflanzenschutz
}

\subsection{Tierhaltung}

Die Übertragung von Antibiotika-Resistenzen vom Tier auf den Menschen trägt zur aktuellen Resistenz-Problematik bei. ${ }^{80}$ Ein besonderes Problem stellen gramnegative ESBL-bildende Bakterien dar (s. auch Kapitel 2.2). Resistente Bakterien können den Menschen über Lebensmittel oder über den direkten Kontakten mit Tieren erreichen. Die Übertragung von MRSAIsolaten vom Sequenztyp ST398 vor allem von Schweinen auf Menschen wurde mehrfach nachgewiesen. ${ }^{81}$ Der horizontale Gentransfer zwischen Keimen von Tieren und von Menschen spielt möglicherweise ebenfalls eine Rolle bei der Ausbreitung von Resistenzen.

Grundsätzlich sollten Antibiotika nur in unbedingt notwendigem Umfang Anwendung finden. Generell sollten Antibiotika, wie bereits 2010 in den Leitlinien der Bundestierärztekammer festgelegt, bei Tieren nur therapeutisch eingesetzt werden und - abgesehen von wenigen gerechtfertigten Ausnahmen - nicht zur Prophylaxe. ${ }^{82,83}$ Der Einsatz von antimikrobiell wirksamen Substanzen mit dem Ziel, den Produktionserfolg in der Mast zu steigern, wurde bereits 2006 in der EU verboten.

Antibiotika-Resistenzen, die in der Tierhaltung entstehen, können die Wirksamkeit wichtiger Antibiotika in der Humanmedizin gefährden. Bestimmte Klassen von Antibiotika sollten für die humanmedizinische Anwendung reserviert bleiben. Auch Neuentwicklungen sollten in der Veterinärmedizin möglichst nicht verwendet werden. ${ }^{84}$ Darüber hinaus ist es unerlässlich, tierpathogene Bakterien und Zoonose-Erreger zu über-

80 Bywater RJ \& Casewell MW (2000) beziffern den Beitrag der Veterinärmedizin/ Tierproduktion an der Resistenzproblematik in der Humanmedizin auf unter 4 Prozent. 81 Voss A et al (2005); Wulf M \& Voss A (2008); van Loo l et al (2007); Cuny C et al (2010).

82 Bundestierärztekammer (2010).

83 Eine Ausnahme ist beispielsweise die perioperative Gabe von Antibiotika im Falle von Fremdkörperoperationen bei Hunden, wenn der Darm eröffnet wird.

84 Derzeit gibt es keine veterinärmedizinische Zulassung für Oxazolidinone (Linezolid), zyklische Lipopeptide (Daptomycin), Glycopeptide (Vancomycin, Teicoplanin), Glycylcycline (Tigecyclin), Streptogramine (Synercid - Quinu/ Dalfopristin), Mupirocin. Allerdings können all diese Substanzen im Falle eines Therapienotstands (z. B. bei pan-resistentem Staphylococcus pseudintermedius von Hunden) bei Tieren, die nicht der Lebensmittelgewinnung dienen (Hund/Katze), umgewidmet und dann eingesetzt werden. 
wachen sowie weiterhin Resistenzdaten zu erheben, wie dies seit 2001 in Deutschland im Rahmen des vom Bundesamt für Verbraucherschutz und Lebensmittelsicherheit durchgeführten nationalen Resistenz-Monitoringprogramms GermVet durchgeführt wird.

Durch regelmäßige Fortbildungsmaßnahmen muss auch bei Beschäftigten in der Landwirtschaft und im Lebensmittelbereich das Bewusstsein für Antibiotika-Resistenzen und die Mechanismen ihrer Entstehung und Verbreitung geschärft werden.

Die Auswirkungen des Einsatzes von Antibiotika bei Tieren auf die Entstehung von Resistenzen und die Aufklärung der Übertragungswege sind bereits Thema mehrerer Forschungsverbünde. So untersucht z. B. der Verbund RESET ${ }^{85}$ die Entstehung von Resistenzen am Beispiel von Enterobakterien (Escherichia coli und Salmonella enterica), während der Verbund MedVet-Staph Staphylococcus aureus (MRSA) ${ }^{86}$ untersucht. Dennoch sollte stärker erforscht werden, welchen Einfluss die Gabe von Antibiotika bei Tieren auf die Entstehung von Resistenzen und die Weitergabe von Resistenzfaktoren auf humanpathogene Erreger oder die Übertragung pathogener Bakterien von Nutztieren auf den Menschen haben.

\subsection{Pflanzenschutz}

Einige Antibiotika wie Streptomycin und Oxytetracyclin werden im Obstund Gemüseanbau gegen pflanzenpathogene Bakterien eingesetzt. Streptomycin wird in vielen Ländern der EU und außerhalb Europas zur Vorbeugung des Feuerbrands verwendet, der durch das Bakterium Erwinia amylovora verursacht wird. Dadurch wird der Ausbruch der Krankheit zwar effektiv verhindert, die Entstehung von Antibiotika-Resistenzen jedoch gefördert. Auf mobilen genetischen Elementen fanden sich Streptomycin-Resistenzgene, die für ein Phosphotransferase-Enzym (StrA, StrB) kodieren. Nachweislich gibt es die gleichen Gene in 17 Arten von Umweltbakterien und Krankheitserregern. ${ }^{87}$ Obwohl die Menge der eingesetzten Antibiotika im Pflanzenanbau weitaus geringer ist als in der Humanmedizin und in der Tiermedizin, sollte jede großflächige Ausbringung von Antibiotika vermieden und durch andere infektionsprophylaktische Maßnahmen ersetzt werden.

85 http://www.reset-verbund.de/ (abgerufen am: 13. April 2012).

86 http://www.medvetstaph.net/index.html (abgerufen am: 13. April 2012).

87 McGhee GC et al (2011). 